\title{
Gradient Theory and Its Applications to Stability Analysis of Materials and Structures
}

\author{
J. NING and E.C. AIFANTIS* \\ Center for Mechanics of Materials and Instabilities, Michigan Tech., Houghton, MI 49931, USA
}

\begin{abstract}
Higher-order strain gradient and internal variable theories are used to investigate material instability caused by geometry softening (necking) and material softening (shear banding). The effects of anisotropic hardening/softening behavior and void density are taken into account by incorporating appropriate internal variables into the constitutive equations. Theoretical predictions compare well with corresponding experimental data.
\end{abstract}

\section{Introduction and Background}

Deformation inhomogeneity is an inherent property of materials. It is responsible for the occurrence of deformation localization, instability, fracture and failure in a variety of materials ranging from metals and polymers to ceramics and rocks. In order to describe the development of plastic inhomogeneity and associated plastic instabilities, a gradient theory of plasticity was developed by Aifantis [1-4] and co-workers [5-9] by utilizing a gradient-dependent expression for the flow stress. The simplest form of this gradient-dependent flow stress reads

*. Also: Aristotle University of Thessaloniki, Thessaloniki 54006, GREECE 


$$
\tau=\kappa(\gamma)-c \nabla^{2} \gamma
$$

where $(\tau, \gamma)$ are the equivalent stress and strain invariants, $\kappa(\gamma)$ is the usual homogeneous part of the flow stress and the gradient coefficient $c$ measures the heterogeneous or nonlocal character of the pertinent hardening/softening mechanisms. It has been argued (see, for example, [9]) that the sign of c may be positive or negative depending on the deformation state (positive for softening and negative for hardening). A more general form of the gradient-dependent flow stress is given by [1]

$$
\tau=\kappa(\gamma)-c_{1} \mid \nabla \gamma^{2}-c_{2} \nabla^{2} \gamma
$$

where $c_{1}$ and $c_{2}$ are the first and second order strain gradient coefficients.

A geometric justification of this formula can be provided in terms of the Bridgman's factor for a necked tensile bar. According to Bridgman's [10] analysis for the stress distribution in the neck, the true stress $\sigma$ at the surface of the specimen reads

$$
\sigma \approx \sigma /\left(1+\frac{1}{2} \frac{a}{R}\right)
$$

where $a$ is the radius of the neck, and $\sigma$ is the average stress over the crosssection of the specimen, and $R$ denotes the radius of curvature of the neck (see Fig. 1) defined by the relationship

$$
R^{-1}=\frac{d^{2} \rho}{d x^{2}},
$$

where $\rho$ denotes the radius of the specimen. According to the definition of strain

$$
\varepsilon=2 \ln \left(\rho_{0} / \rho\right)
$$

we obtain upon differentiation relation 


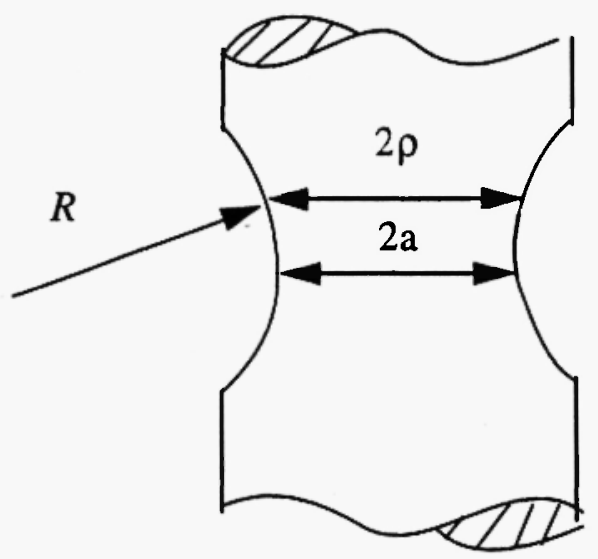

Fig. 1 Necking in uniaxial tension bar.

$$
\frac{d^{2} \rho}{d x^{2}}=4 \rho\left(\frac{d \varepsilon}{d x}\right)^{2}-2 \rho \frac{d^{2} \varepsilon}{d x^{2}}
$$

Then, eqns. (3), (4) and (6), together with the usually assumed constitutive equation for the (average) true stress $\delta=\kappa(\varepsilon)$ gives the following expression for the local (Cauchy) stress

$$
\sigma \approx \kappa(\varepsilon)\left\{1-2 a^{2}\left(\frac{d \varepsilon}{d x}\right)^{2}+a^{2} \frac{d^{2} \varepsilon}{d x^{2}}\right\},
$$

It is noted that a relation of this form was obtained by Coleman [11] by reducing a 3D strain state for a long slender cylindrical fiber made of a nonlinear elastic material to a 1D one. Coleman used the existence of a free energy density function and related thermodynamics arguments to obtain in (2) the restriction $c_{1}(\lambda)=-c_{2}^{\prime}(\lambda) / 2$. This is, in our opinion, a very special conclusion.

The nature of the strain gradient coefficient $c$ in (1) has been discussed on the basis of certain microscopic arguments. These estimates for the coefficient $c$ depend on the scale of interest and the particular deformation mechanisms involved. For granular materials, a Taylor series expansion for the average strain 
has led [6-7] to the conclusion that the coefficient $c$ scales with the grain size $R$ via a relationship of the form

$$
c=C R^{2},
$$

where the magnitude of the "scaling" coefficient $C$ is proportional to the plastic modulus and a geometric factor ( $1 / 8$ for $2-\mathrm{D}$ and $1 / 10$ for $3-\mathrm{D}$ ). The Taylor series expansion argument for the average strain was recently utilized by Zbib [12] within a self-consistent framework for particulate composites to obtain a relationship like (3) but with $C$ proportional to the matrix shear modulus. A more complete discussion was provided by Ning and Aifantis [9] which also arrives at (3) but with c depending on both elastic and plastic moduli (see also [4]).

While various aspects of strain gradient theories can be found in [1-4] and references quoted therein, the focus in this paper is to discuss the implications of gradient effects on necking and shear banding by using either (1) or an alternative gradient formulation based on internal variables with diffusive effects [4].

\section{Higher-Order Strain Gradient Theory}

A direct application of the strain gradient theory (1) or (2) can be found in the analysis of material instability associated with the phenomena of shear banding (material softening) and necking (geometry softening).

\subsection{Shear Banding}

This problem has been analyzed by various investigators (see, for example, in the references of [1-4]) and we only review here the main results. For a gradient-dependent rigid plastic material, the appropriate constitutive equations read

$$
\sigma=-p \underline{I}+2 \mu D ; \mu=\tau / \gamma,
$$

where the flow stress $\tau$ is described by (1).

The initiation of instability can be determined by using a standard linear stability 
analysis. The velocity field that admits representation is thus assumed to be of the form

$$
\underline{v}=L^{\circ} \underline{x}+\varepsilon v^{\circ} \underline{v} \exp (i q z+\omega t)
$$

with $z=\underset{\sim}{n} \cdot \underset{\sim}{x}$, and $\left(L^{\circ}, v^{\circ}\right)$ being constants. $(\underline{n}, \underline{v})$ denote, respectively, unit vectors normal and parallel to the band. For plane deformation, the linear stability analysis of (9) and (10) leads [5] to the following expressing expression for the growth rate $\omega$ of exponential fluctuations $\left(\sim e^{i q x+\omega t} ; \mathrm{q}=\right.$ wave number, $\mathrm{x}=$ coordinate normal to the band)

$$
\omega=-\frac{h+c q^{2}}{\mu f(\theta)} ; h=\kappa^{\prime}(\gamma)
$$

where $2 \pi / q$ is the wavelength of the fluctuation and $\theta$ denotes the angle of shear band inclination. It turns out that from (9) and (10) that $f(\theta)=\cot ^{2} 2 \theta$ for plane strain deformation, and $f(\theta)=-1+16 /\left(3\left(5+2 \cos 2 \theta-3 \cos ^{2} 2 \theta\right)\right)$ for plane stress deformation. ${ }^{*}$. By maximizing $\omega$ we obtain the critical condition $\theta=\pi / 4$, $q_{c}=0, h_{c}=0$ for the condition of emergence of shear bands. However, if a fourth order strain gradient term is added into the constitutive equation (1), the preferred wavenumber $q_{c}$ and the critical shear modulus $h_{c}$ are not equal to zero but depend on the gradient coefficients [5].

After the shear band is formed at an angle $\theta$ with respect to the $\mathrm{x}$ axis, we

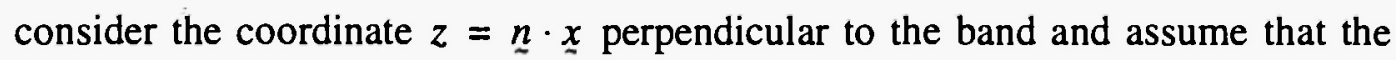
deformation field is of the form of simple shear in the direction of the band. It then turns out that the governing equation reads

$$
c \gamma_{22}=\kappa(\gamma)-\kappa_{0}
$$

where the homogeneous portion of the flow stress $\kappa(\gamma)$ is a softening type curve with a single local maximum or a non-convex curve with a single loop. By utilizing the technique of Aifantis and Serrin [13] we find the explicit solution 


$$
z=\bar{z}-\int_{\gamma(z)}^{\gamma(z)}\left\{\frac{2}{r}\left[\int_{\gamma_{i}}^{\gamma}\left(\kappa(\gamma)-\kappa_{0}\right) d \gamma\right]^{-1 / 2}\right\} d \gamma,
$$

where $z$ is an arbitrary point and $\gamma_{1}$ is the shear strain at $z= \pm \infty$. The profile of $\gamma(z)$ according to (13) is a smooth and symmetric curve having a maximum at the center of the shear band. Following the method developed in [13] and the constitutive relation

$$
\tau=\tau_{m}-\alpha\left(\gamma-\gamma_{m}\right)^{2}
$$

where $\tau_{m}, \alpha$ and $\gamma_{m}$ are constants, the width $w$ of shear band can be found as follows

$$
w=2.924 \sqrt[i]{\frac{\hat{c}}{2 \ddot{u}}} \delta^{-1 / 4},
$$

with the definition

$$
\delta=\left(\gamma_{m}-\gamma_{1}\right)^{2}
$$

The effects of imperfections and material parameters has also been discussed in detail by using numerical solution (Zbib and Aifantis [5, 14]).

* For a constitutive equation of the form $\underset{\sim}{S}=-p \underline{I}+2 \mu \underset{\sim}{D}$, with $\mu=\tau / \gamma$, $\tau=\sqrt{S^{\prime \prime} \cdot \underline{S}^{\prime} / 2}, \gamma=\sqrt{2 \underline{D} \cdot \underline{D}}$ and $\left(p, \underline{S}^{\prime \prime}\right)$ denoting the pressure and the deviatoric part of Cauchy stress $\underset{\sim}{S}$, the linear stability analysis gives the onset

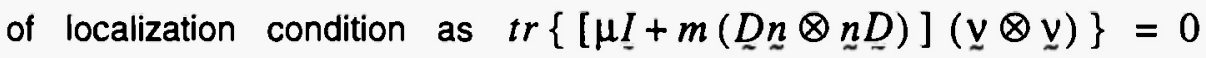
where $m=4\left(h-c q^{2}-\mu \omega\right) / \omega \gamma$. For a plane deformation, the unit vectors $\underline{n}$ and $\underline{\mathrm{v}}$ are given by $\left(n_{1}, n_{2}\right)=\left(-\mathrm{v}_{2}, \mathrm{v}_{1}\right)=(-\sin \theta, \cos \theta)$. In particular, we have $D_{11}=-D_{22}, D_{33}=0$ and $\dot{\gamma}=2 D_{11}$ for plane strain; $S_{11}^{\prime}=-S_{22}^{\prime}, S_{33}^{\prime}=0$ and $\tau=S_{11}$ for plane stress. On substituting these relations together with the above constitutive equation into the onset of localization condition, we can obtain eqn. (11) with $f(\theta)=\cot ^{2} 2 \theta$ for plane strain deformation, and $f(\theta)=-1+16 /\left(3\left(5+2 \cos 2 \theta-3 \cos ^{2} 2 \theta\right)\right)$ for plane stress deformation. 


\subsection{Necking}

An incompressible and rigid-plastic solid tension specimen with the length $2 L^{\infty}$ in the initial undeformed state is considered. A Largrangian, one-dimensional reference system is adopted where material points are labelled by their convected coordinates $x$ along the axis of the specimen in its initial reference configuration. The origin of the $x$-axis is located at the mid-section of the specimen. Because of the symmetry of the problem, only one half of the specimen is analyzed.

The axial stretch $\lambda$ and true (logarithmic) strain $\varepsilon$ are defined by

$$
\lambda=1+\frac{\partial u}{\partial x} ; \varepsilon=\ln \lambda .
$$

The momentum balance equation reads

$$
\rho_{0} A^{0}(x) \frac{\partial v}{\partial t}=\frac{\partial}{\partial x}[\sigma A(x, t)]
$$

where $\rho_{0}, \sigma, v$ and $A(x, t)$ denote, respectively, the material density, stress, velocity and the current section area of the specimen at time $t$. It is noted that $A(x, 0)=A^{0}(x)$.

The continuity equation (incompressibility) reads

$$
\frac{\partial \varepsilon}{\partial t}=-\frac{A}{A} .
$$

The compatibility equation between $v$ and $\varepsilon$ is given by

$$
\frac{\partial v}{\partial x}=e^{\varepsilon} \frac{\partial \varepsilon}{\partial i}
$$

The boundary and initial conditions are usually assumed to be of the form

$$
\begin{gathered}
v(0, t)=0 ; v\left(L_{0}, t\right)=V ., \\
v(x, 0)=V x / L_{0} ; \varepsilon(x, 0)=0 .
\end{gathered}
$$

Two different types of constitutive equations based on (1) are employed in the following analysis to consider the effects of different material hardening behavior: 


\section{Power hardening}

$$
\sigma_{e}=K \varepsilon^{n}+C_{1} \frac{d^{2} \varepsilon}{d x^{2}}
$$

where $\sigma_{e}=\sigma-\alpha, C_{1}$ is the strain gradient coefficient, and $\alpha$ is the back stress with evolving with the rule (Prager type hardening)

$$
\dot{\alpha}=g \dot{\varepsilon},
$$

where $g$ is a anisotropic hardening material parameter.

Saturated hardening

$$
\sigma_{e}=\sigma_{\mathrm{s}}\left[a+(1-a) e^{-b \varepsilon}\right]+C_{1} \frac{a^{2} \varepsilon}{d x^{2}}
$$

where $A, b$ and $C_{1}$ denote material isotropic hardening material parameters and the strain gradient coefficient, respectively. The corresponding back stress is assumed to obey an Armstrong-Frederick's hardening rule

$$
\dot{\alpha}=g_{1} \dot{\varepsilon}-g_{2}|\dot{\varepsilon}| \alpha,
$$

where $g_{1}$ and $g_{2}$ are anisotropic hardening material parameters.

The linear stability analysis for power hardening material based on equations (14)-(20) gives the following condition for the initiation of necking:

$$
\varepsilon_{0}=n \div \frac{\left(1-\varepsilon_{0}\right) g-C_{1} q^{2}}{K}\left(\varepsilon_{0}\right)^{1-n} .
$$

When $C_{1}=g=0$, the above inequality reduces to the well-known Considere criterion

$$
\varepsilon_{0}>n
$$

Linear stability analysis provides information for the very early development of small non-uniformities of deformation; however, it cannot be used for estimating 
the influence of imperfections or material parameters on the amount of attainable strain [15]. Therefore, the "nonlinear long-wavelength" method is used in the following to investigate the evolution of necking.

For the emergence of necking, an initial geometric imperfection is usually introduced in the region $\left[0, L_{0}\right]$, where $L_{0}<L^{-*}$. The specimen thus consists of two regions: the "bulk" (defect-free) region with a homogeneously distributed strain $\varepsilon_{0}$ and cross-sectional area $A_{0}(t)$, and the "necked" region with the cross-section area $A(x, 0)$ varying along its axis $x$ in the undeformed state according to the relation

$$
A(x, 0)=A_{0}(0)\left(1-\eta \cos \left(\frac{\pi x}{2 L_{0}}\right)\right),
$$

where $\eta(0<\eta \ll 1)$ denotes the amplitude of the imperfection and $A_{0}(0)$ denotes the cross-sectional area in the bulk region in the undeformed state.

In view of the assumption of incompressibility, the true strains in the necked and bulk regions are expressed by the relations

$$
\varepsilon=-\ln \frac{A(x, t)}{A(x, 0)} ; \quad \varepsilon_{0}=-\ln \frac{A_{0}(t)}{A_{0}(0)} .
$$

The spatial distribution of strain in the necked region is obtained based on (18)(26) and (29)-(30) as follows

$$
\left.\tau_{i} \frac{d^{2} \varepsilon}{d \bar{x}^{2}}=\frac{\bar{g} \varepsilon_{0}+\varepsilon_{0}^{n}}{[1-\eta \cos (\pi \bar{x} / 2)]} e^{\varepsilon-\varepsilon_{0}}-i \bar{g} \varepsilon+\varepsilon^{n}\right)
$$

for power hardening material, and

$$
\begin{aligned}
C^{\prime}{ }_{1} \frac{d^{2} \varepsilon}{d \bar{x}^{2}} & =\frac{\left[A+(1-A) e^{-h \varepsilon_{0}}\right]+\bar{g}_{1}\left(1-e^{-g_{2} \varepsilon_{0}}\right) / g_{2}}{[1-\eta \cos (\pi x / 2)]} \epsilon^{\varepsilon-\varepsilon_{0}} \\
- & {\left[A+(1-A) e^{-\overline{i . z}}\right]-\bar{g}_{1}\left(1-e^{-g_{2}{ }^{2}}\right) / g_{2} }
\end{aligned}
$$

for saturated hardening material, where the dimensionless variables are defined 
by

$$
\bar{x}=\frac{x}{L_{0}}, \quad \bar{g}=\frac{g}{K}, \quad \bar{g}_{1}=\frac{g_{1}}{\sigma_{s}}, \quad \bar{C}_{1}=\frac{C_{1}}{K L_{0}^{2}}, \quad C_{1}=\frac{C_{1}}{\sigma_{s} L_{0}^{2}} .
$$

In the calculation, the values of the parameters are taken as $\eta=0.05$, $a=2, b=1, g=g_{1}=2, g_{2}=5, C_{1}=0.08, C_{1}=0.06$ which are within the rang of modeling deformation behavior of structural steels.

The true stress-strain relations and the corresponding engineering stress-strain relations in the necked and the bulk regions are shown in Figs. $2 \mathrm{a}$ and $2 \mathrm{~b}$ for the power hardening material. It is observed that the strain-localization will lead to the true stress-strain curve in the necked region upturn as shown by Hutchinson and Neale [15]. However, the corresponding engineering stress-strain curve is in softening regime after the initiation of localized necking. The results indicate that the gradient approach can model material behavior both in the pre- and postdeformation localization regimes.

The theoretical predictions of strain localization behavior of 70-30 Brass and Phosphor Bronze in uniaxial tension are compared with the corresponding experimental data [16] in Figs. 3a and 3b. The experimental data in [16] show that 70-30 Brass is a power hardening material and Phosphor Bronze is a saturated hardening material. According to the stress-strain curves of these two materials given in [17], the material parameters in the corresponding constitutive equations are identified as $K=51(k s i), g=60(k s i), n=0.37, \widehat{c}_{1}=0.00026$ for 70 30 Brass, and $A=15.19, b=0.16, g_{1}=2148(k s i), g_{2}=103$, and $C_{1}=0.023$ for Phosphor Bronze. The results in these two figures show the excellent agreement between the theoretical predictions and the corresponding experimental data. 


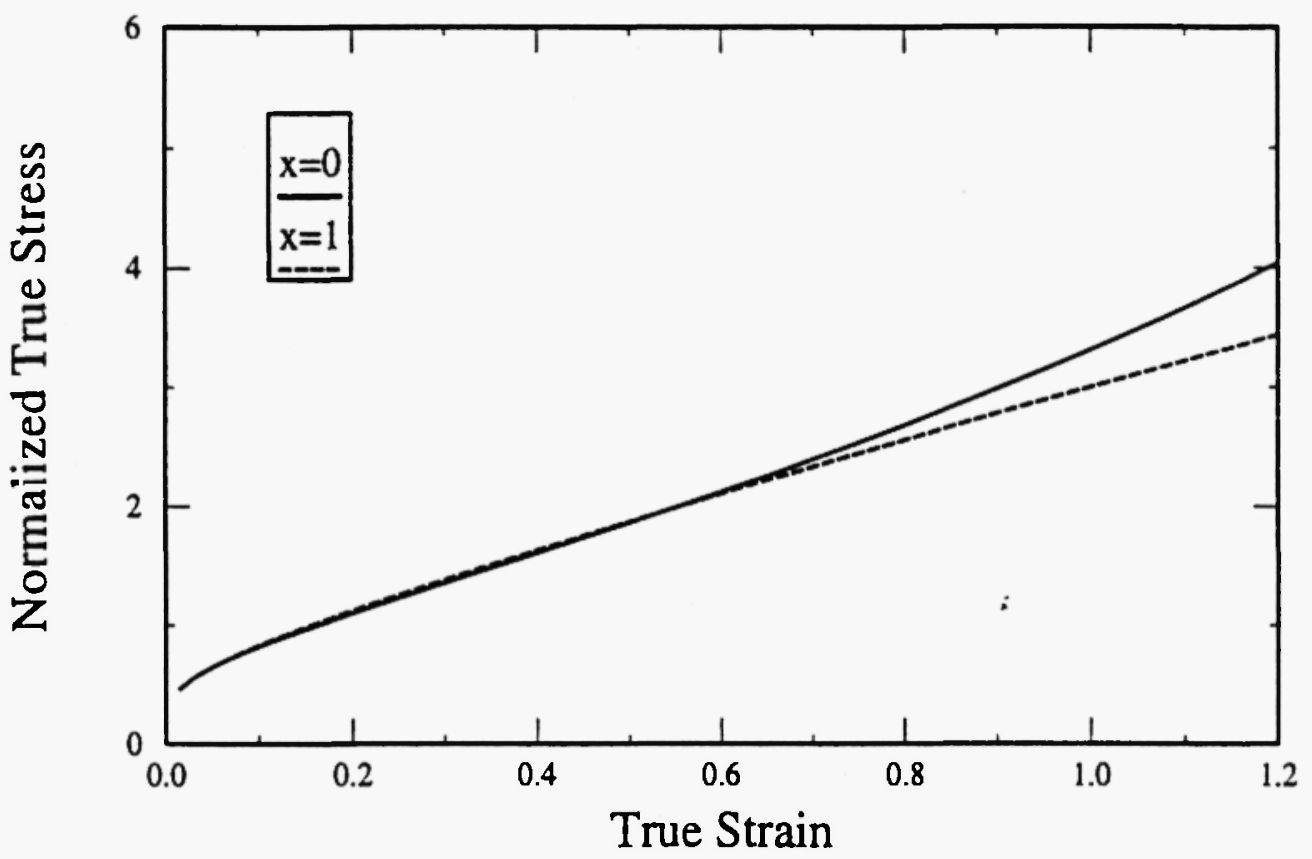

Fig. 2a Normalized true stress $(\sigma / K)$-strain relations within and outside the neck.

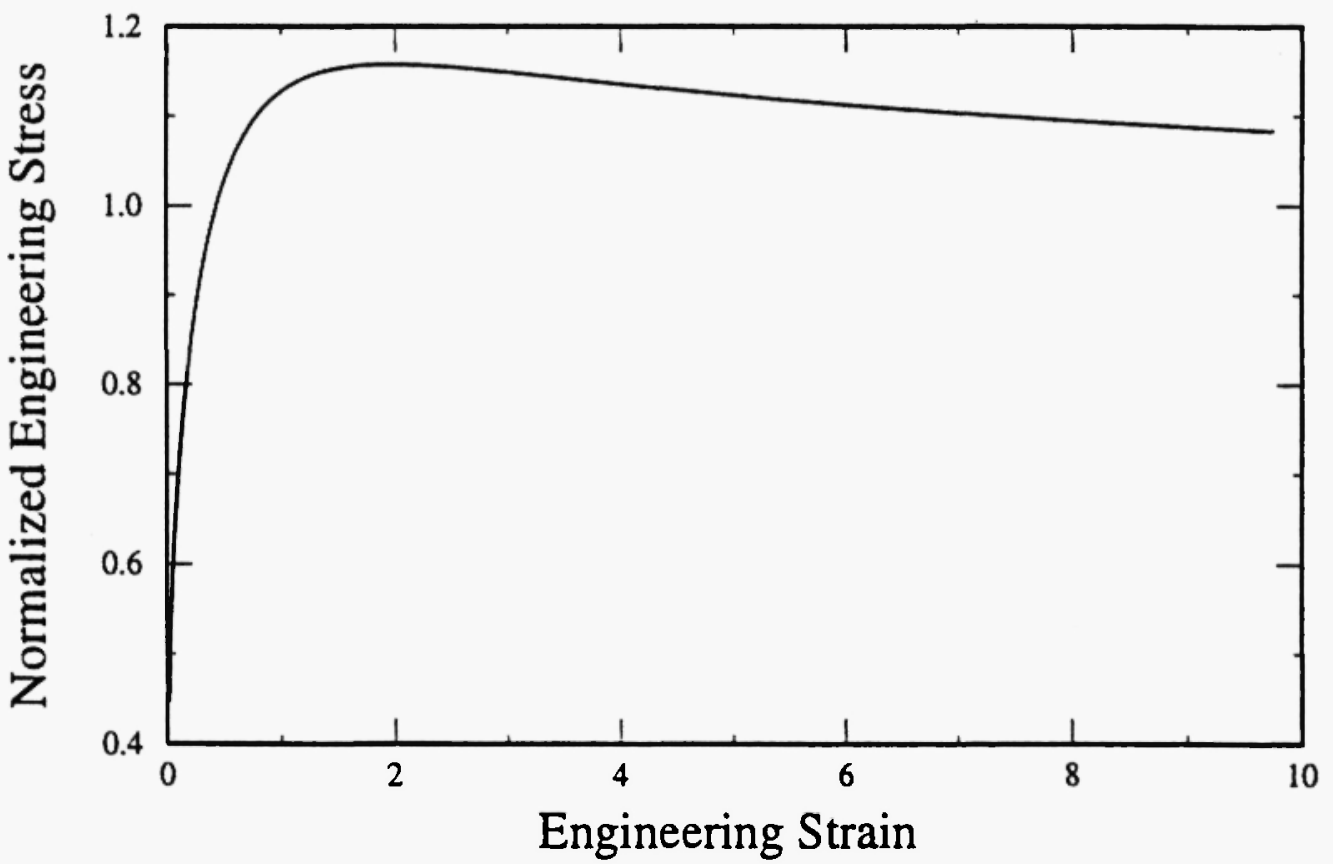

Fig. 2b Normalized engineering stress $(P / A(0,0) K)$-strain relation at the location $\bar{x}=0$. 


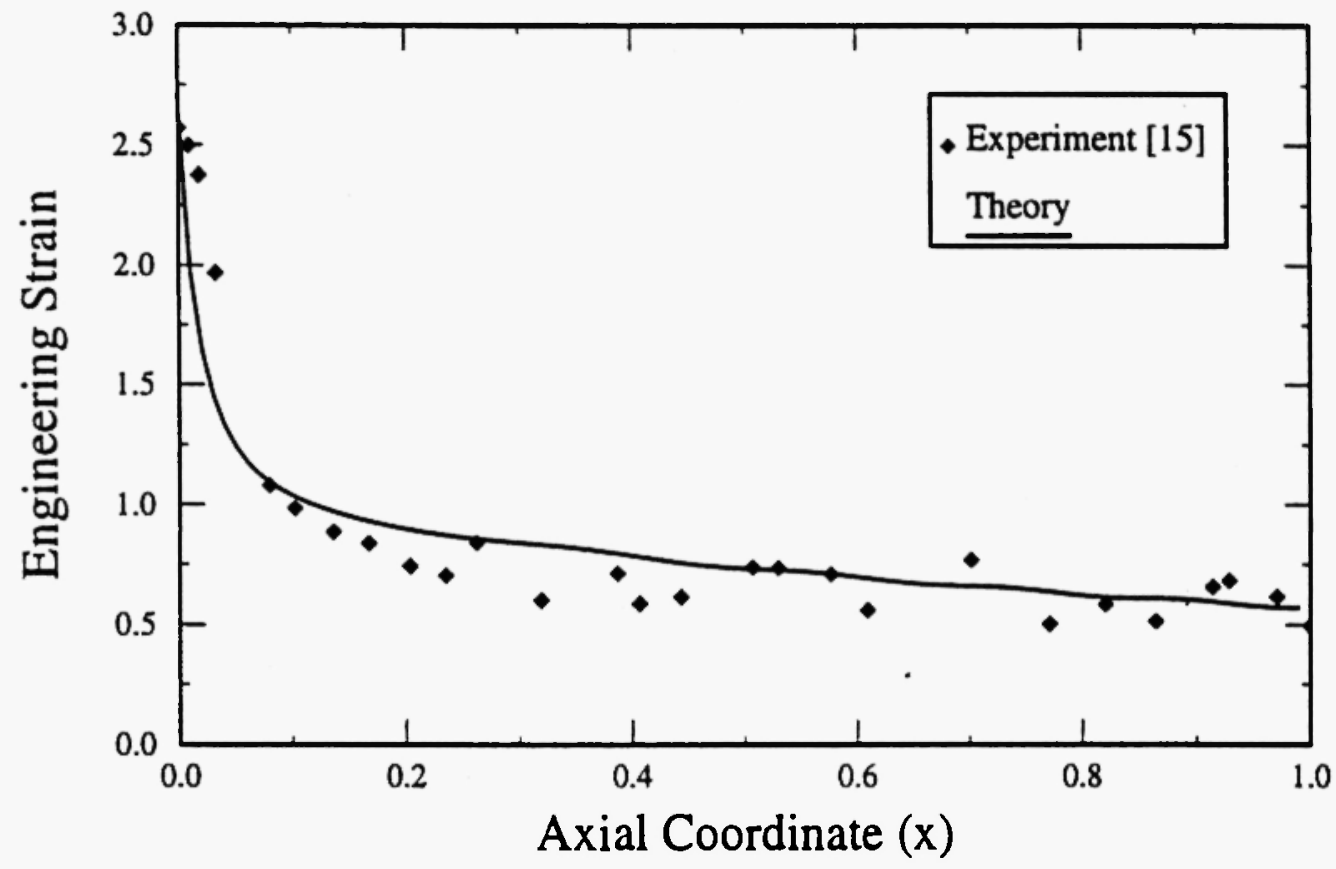

Fig. 3a Comparison between the present theoretical predictions for the strain distribution of 70-30 Brass in uniaxial tension and corresponding experimental data.

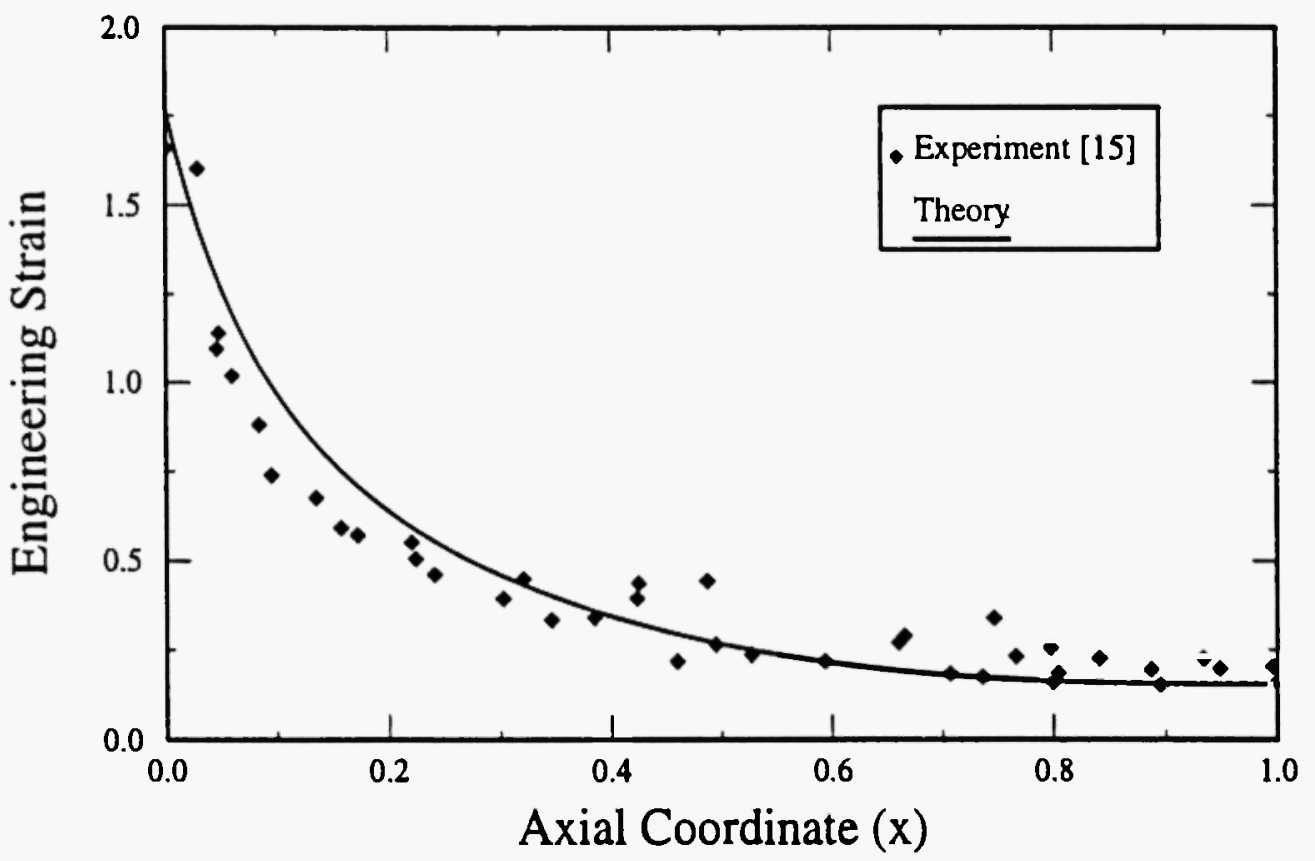

Fig. 3b Comparison between the present theoretical predictions for the strain distribution of Phosphor Bronze in uniaxial tension and corresponding experimental data. 


\section{Higher-Order Internal Variable Theory}

Since the plastic strain is not a state variable, it is more appropriate to use a formulation based on internal variables which are directly related to the inhomogeneous evolution of microstructure [3]. In general, the yield condition $F$ for a plastically deformed material reads

$$
F\left(\tau, p, R, \theta, q_{n}\right)=0
$$

where $(\tau, p)$ denote respectively the shear and hydrostatic stress intensity, $R$ denotes flow stress representing the size of the yield surface. $q_{n}(n=1,2, \ldots)$ are a set of internal variables describing the evolution of microstructure in the plastic deformation and $\theta$ denotes the temperature. According to (1), the flow stress reads

$$
R=\kappa(\gamma, \gamma, \theta, p)+C_{1} \nabla^{2} \gamma
$$

where $C_{1}=-c$ denotes the strain gradient coefficient.

The evolution equations for the internal variables $q_{n}$ with respect to an appropriate objective rate should be described by the "complete" balance laws to account for diffusive effects due to spatial patterning and self-organization of microstructure, i.e.

$$
\dot{q}_{n}=\underset{\sim}{f}\left(\underline{D}, \theta, \underline{q}_{n}\right)+c_{n} \nabla^{2} q_{n},
$$

where $\underset{\sim}{D}$ denotes the plastic strain rate and $c_{n}$ denote appropriate gradient coefficients which may be functions of the equivalent strain and its rate.

For a rigid-plastic and strain rate independent material, the yield condition is assumed to be of the form

$$
\tau(\underline{S}, \underline{\alpha})-R=0,
$$

where the equivalent effective shear stress $\tau$ is defined by $\tau=\sqrt{\frac{1}{2}(\underline{S}-\underline{\alpha}) \cdot(\underline{S}-\underline{\alpha})}$.

with the dot denoting inner product, $\underline{S}$ being the deviatoric part of Cauchy stress $\sigma$, and the internal variable $\underset{\alpha}{ }$ is a second-order deviatoric tensor (back stress) 
modeling anisotropic deformation behavior.

The evolution equation for the back stress can be expressed, in accordance with (36), as

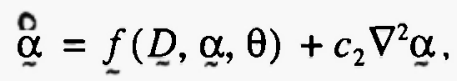

where the superimposed " $。$ " denotes an appropriate corotational rate [3].

For a porous material, an appropriate and convenient yield condition is given by [18]

$$
\tau^{2}=\sigma_{y}^{2}\left\{1+(r \varphi)^{2}-2 r \varphi \cos h\left(3 \sigma_{m} / 2 \sigma_{b}\right)\right\}
$$

where $r=1.5$ and $\tau^{2}=\frac{3}{2} S_{i j} S_{i j}, \quad\left(\sigma_{v}, \sigma_{b}\right)$ denote respectively the yield stress and the ultimate strength of the material, and $\left(S_{i}, \sigma_{m}\right)$ denote respectively deviatoric and hydrostatic stress. The porosity of the material is denoted by $\varphi$.

The evolution of the porosity $\varphi$ is a result of the "growth" and "nucleation" of the porosity:

$$
\dot{\varphi}=\dot{\varphi}_{\text {growth }}+\dot{\varphi}_{\text {nucleation }}+\dot{\varphi}_{\text {coalescence }},
$$

where $\dot{\varphi}_{\text {growth }}+\dot{\varphi}_{\text {coulescence }}$ is resulting from the action of the plastic volumetric strain rate and $\dot{\varphi}_{\text {coalescence }}$ is assumed here to be resulting from diffusion effects associated with rapid void motion. Therefore the "complete" evolution equation for the porosity is taken to be as

$$
\dot{\varphi}=(1-\varphi) \dot{\varepsilon}_{k k}^{p}+c \nabla^{2} \varphi
$$

where $c$ is an appropriate gradient coefficient. 


\subsection{Shear Banding in Anisotropic Hardening/Softening Materials}

Consider a thin layer of constant width $h$ in the direction $y$ which extends to infinity in the directions $x$ and $z$. Assume that all displacements in the directions $y$ and $z$ as well as their derivative with respective to $x$ and $z$ are zero. Then, the only non-vanishing component of the velocity $v$ is in the direction $x$ and given by

$$
v=v(y, t)
$$

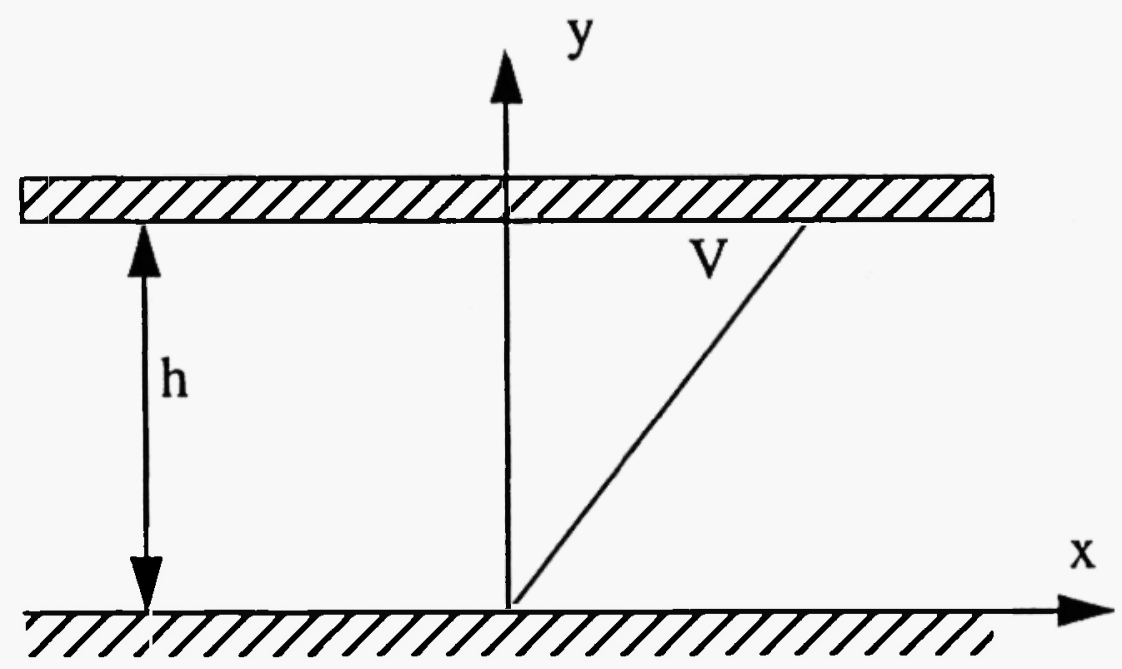

Fig. 4 Shearing thin layer

with the corresponding boundary conditions being

$$
v(0, t)=0 ; v(h, t)=V(\text { for } t>0)
$$

where $V$ denotes the velocity at the boundary.

The momentum balance equation now reads

$$
\rho_{0} \frac{\partial v}{\partial t}=\frac{\partial \tau}{\partial y},
$$

where $\tau$ is the shear stress and $\rho_{0}$ the which is regarded as a constant in the 
following analysis.

If by $\gamma$ we denote the relevant shear strain, the kinematic compatibility equation reads

$$
\frac{\partial \gamma}{\partial t}=\dot{\gamma}=\frac{\partial v}{\partial y}
$$

which upon combination with (44) gives

$$
\rho_{0} \frac{\partial \gamma}{\partial t}=\frac{\partial^{2} \tau}{\partial y^{2}}
$$

The constitutive equation is assumed to be of the form

$$
\tau=K \gamma^{n}+\alpha+C_{1} \frac{\partial^{2} \gamma}{\partial y^{2}}
$$

with the evolution equation of the back stress $\alpha$ given by (38) with $c_{2}=C_{2} \dot{\gamma}$

$$
\frac{\partial \alpha}{\partial t}=\beta \dot{\gamma}+C_{2} \dot{\gamma} \frac{\partial^{2} \alpha}{\partial y^{2}},
$$

where $\beta$ denotes the anisotropic hardening/softening modulus of the material and $C_{2}$ is a constant.

\section{Linear Stability Analysis}

By means of linear stability analysis for eqns. (45)-(48), the characteristic equation for the case of quasi-static deformation is obtained as

$$
\omega+\left\{\frac{1}{\gamma_{0}}+\frac{C_{2} q^{2}\left(h-\beta-C_{1} q^{2}\right)}{h-C_{1} q^{2}}\right\} \because n=0
$$


where $h=n K \gamma_{0}^{n-1}+\beta$ is the tangential modulus of the true stress-strain curve and $\omega$ measures, as usual, the exponential growth of strain fluctuations.

The deformation localization criterion thus reads

$$
\frac{1}{\gamma_{0}}+\frac{C_{2} q^{2}\left(h-\beta-C_{1} q^{2}\right)}{h-C_{1} q^{2}} \leq 0
$$

When the left side of inequality (50) is equal to $-e$ where $e$ is a positive number, the corresponding deformation localization is commonly referred to as effective $e$ instability [19], and it provides a qualitative picture of the nonlinear postinstability situation. The preferred wave number $q_{c}$ is attained by maximizing $h$ :

$$
h_{c}=\max \left\{h\left(q, \gamma_{0}, e\right)\right\} \text {. }
$$

From (50) and (51), we can find that

$$
\begin{gathered}
q_{c}^{2}=-\frac{1+e \gamma_{0}}{C_{2} \gamma_{0}}+\frac{1}{C_{2} \gamma_{0}} \sqrt{-\frac{C_{2} \gamma_{0} \beta\left(1+e \gamma_{0}\right)}{C_{1}}}, \\
h_{r}=\left[\sqrt{-\frac{\left(1-e \gamma_{0}\right) C_{1}}{C_{2} \gamma_{0}}}-\sqrt{\beta}\right]^{2},
\end{gathered}
$$

For the case of "absolute instability" $(e=0)$, we have

$$
\begin{gathered}
q_{c}^{2}=-\frac{1}{C_{2} \gamma_{0}}+\frac{1}{C_{2} \gamma_{0}} \sqrt{-\frac{C_{2} \gamma_{0} \beta}{C_{1}}}, \\
h_{r}=\left[\sqrt{-\frac{C_{1}}{C_{2} \gamma_{0}}}-\sqrt{\beta}\right]^{2},
\end{gathered}
$$

For a power hardening material, we can obtain the plastic modulus from (47)

$$
h_{c}=n K \gamma_{0}^{n-1}+\beta \text {, }
$$


which together with equations (54) and (55) can be used to determine the critical strain $\gamma_{0}$.

\section{Non-Linear Numerical Analysis}

For the convenience of numerical analysis, the governing equations are normalized as follows

$$
\begin{gathered}
\bar{\rho} \frac{\partial \bar{v}}{\partial t}=\frac{\partial \tau}{\partial \bar{y}}, \\
\frac{\partial \gamma}{\partial \bar{t}}=\frac{\partial \bar{v}}{\partial \bar{y}}, \\
\tau=\kappa(\gamma)+\alpha, \\
\frac{\partial \bar{u}}{\partial \bar{v}}=\bar{p} \gamma+\bar{c} \frac{\partial^{2} \pi}{\partial \bar{y}^{2}},
\end{gathered}
$$

where the dimensionless parameters are defined by

$$
\begin{aligned}
& t=V t / h, \quad \bar{y}=y / h, \quad \overline{0}=v / V, \quad \bar{\rho}=\rho_{0} V^{2} / \kappa_{0}, \\
& \tau=\tau / \kappa_{0}, \quad \bar{\alpha}=\alpha / \kappa_{0}, \quad \bar{\beta}=\beta / \kappa_{0}, \quad \bar{C}=C / V h .
\end{aligned}
$$

A two-step difference scheme with a predictor and corrector is developed based on the governing equations (57)-(60). An 81 mesh number is used in the calculation. An initial imperfection is assumed in the form of $Q=-0.15$ at the center node to trigger the shear banding. Three types of material behavior are studied below according to the sign of the anisotropic hardening/softening coefficient $\beta$.

a) Anisotropic hardening ( $\beta>0$ ): Figure 5 illustrates the influence of the value of the gradient coefficient for the back stress on the evolution of the shear band. It is observed that a larger value of the coefficient corresponds to a wider shear band. Figure 6 shows the effect of the anisotropic hardening modulus $\bar{\beta}$ on the deforma- 
tion localization. It indicates that this parameter has little influence on the width of the shear band. The above results suggest that the width of the shear band only depends on the gradient coefficient.

Figure 7 presents the localization behavior within the shear band as a function of the normalized time (deformation). With the increase of deformation, the intensity of localization decreases indicating the stabilizing role of anisotropic hardening; in particular, that of the gradient of back stress. This behavior indicates that an initially developed shear band may "heal" as a result of opposing back stress gradient development due to anisotropic hardening. This trend is illustrated in Figure 8. It suggests that the differences between flow stress curves for materials with different gradient coefficients are very small in the case of deformation hardening. In fact, deformation localization is suppressed by the rapidly growing deformation in the neighborhood of an initially developed deformation inhomogeneity so that an eventually homogeneous deformation state sets in.

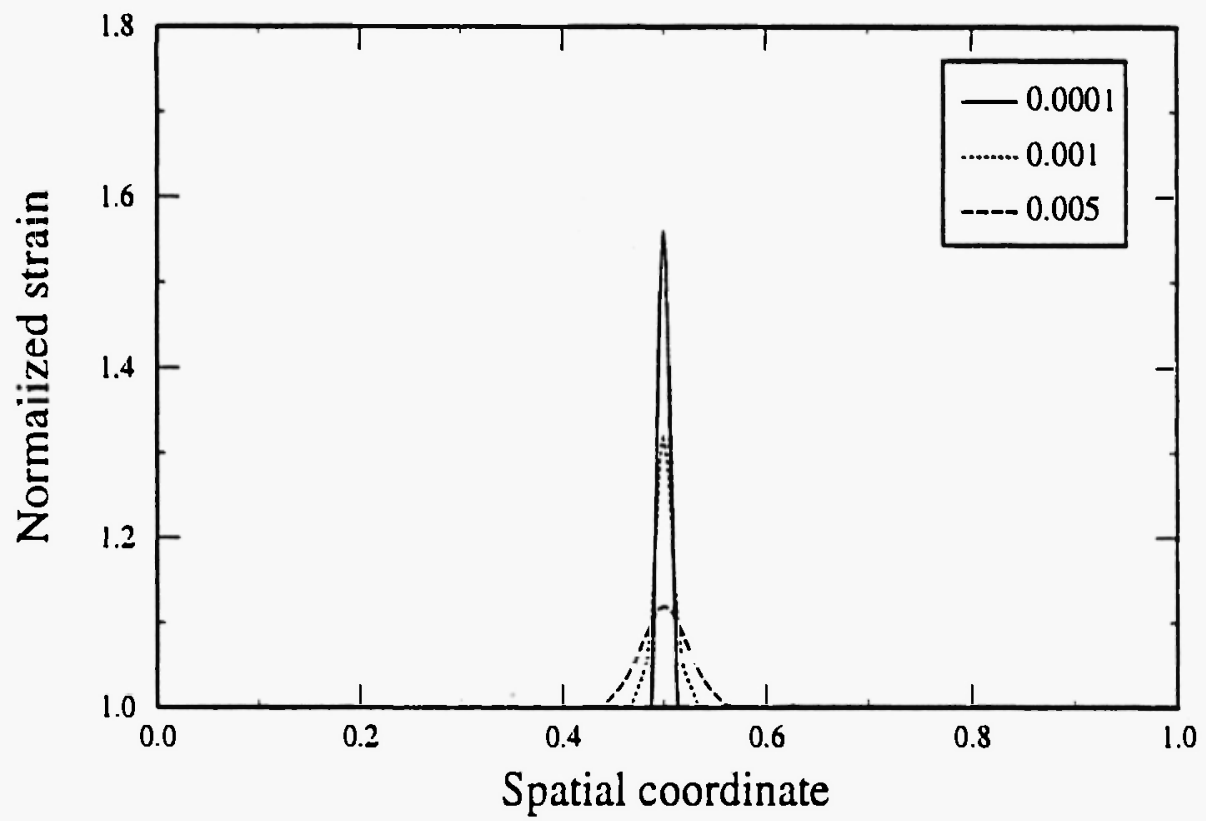

Fig. 5 Effect of the gradient coefficient $\bar{C}$ on the width of shear band. The normalized strain is defined by $\gamma / \gamma_{0}(\beta=1.0, t=0.2)$. 


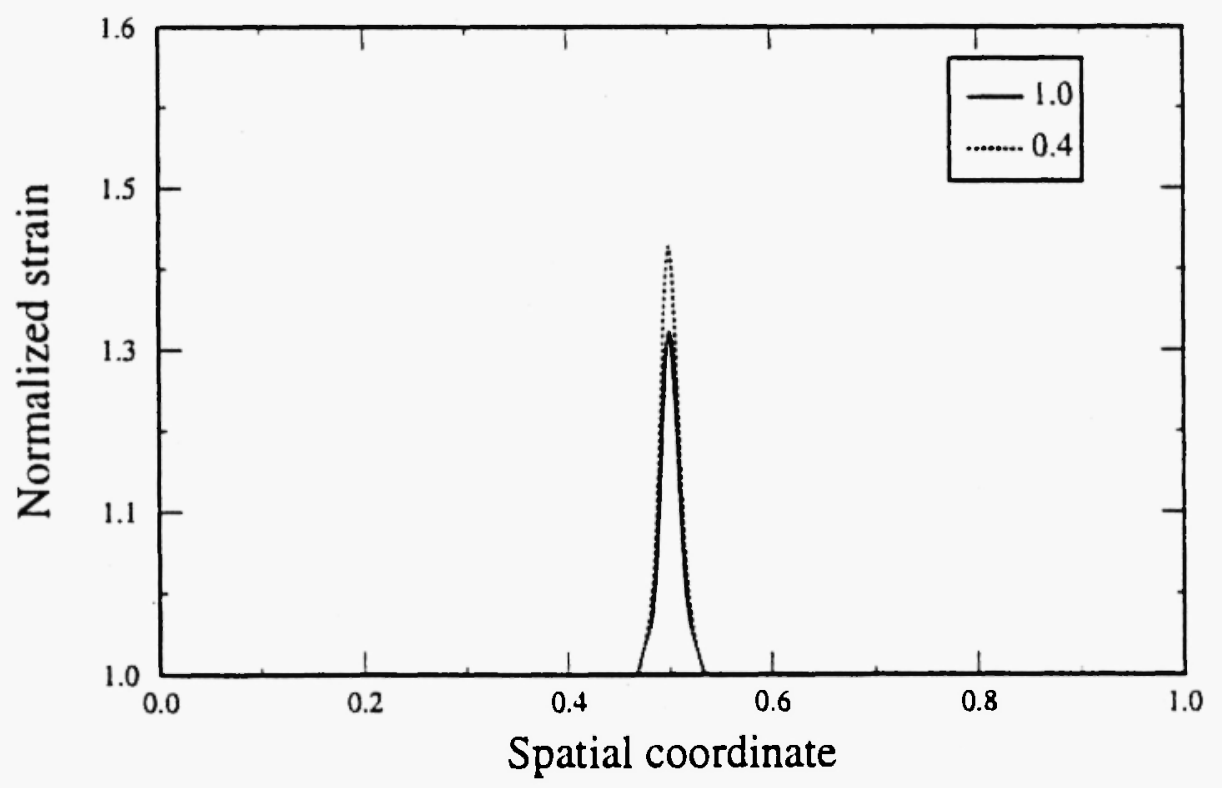

Fig. 6 Effect of the parameter $\bar{\beta}$ on the localization $(t=0.2$ and $C=0.001$ ).

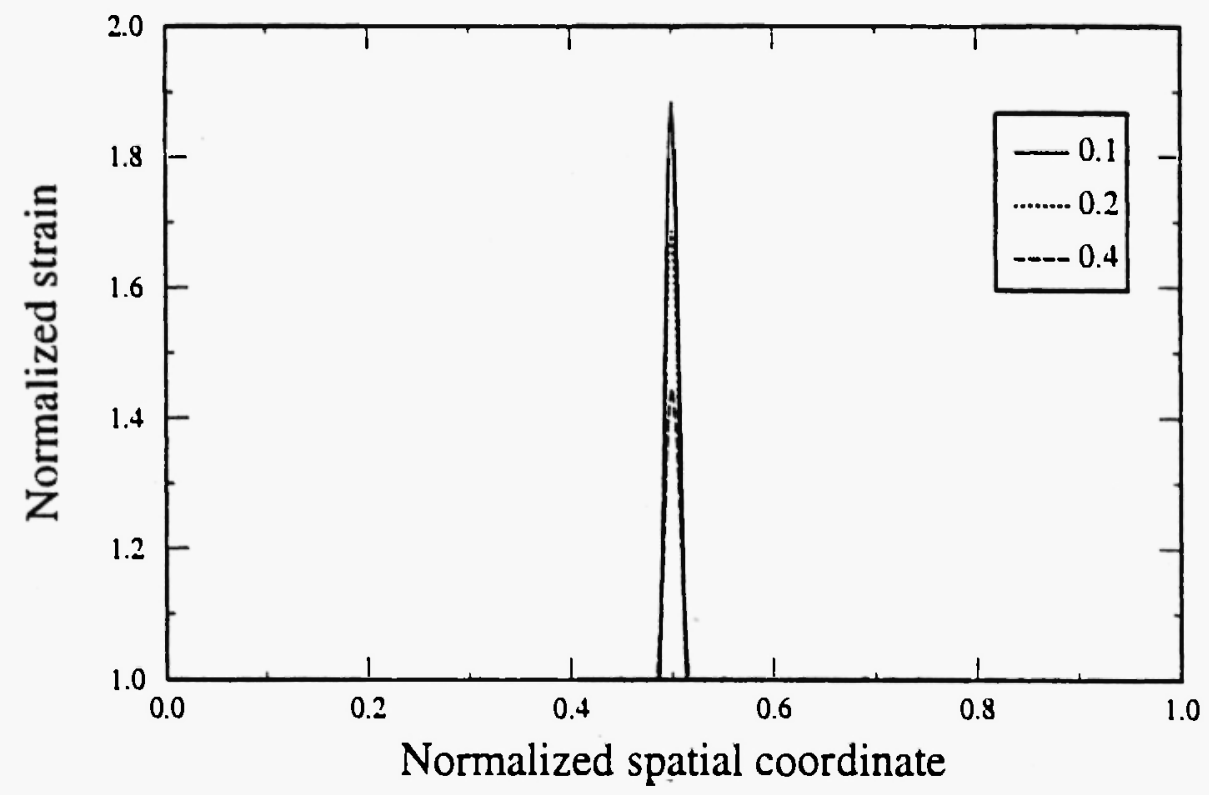

Fig. 7 Evolution of the normalized strain with $t(\bar{\beta}=0.4, C=0.002)$. 


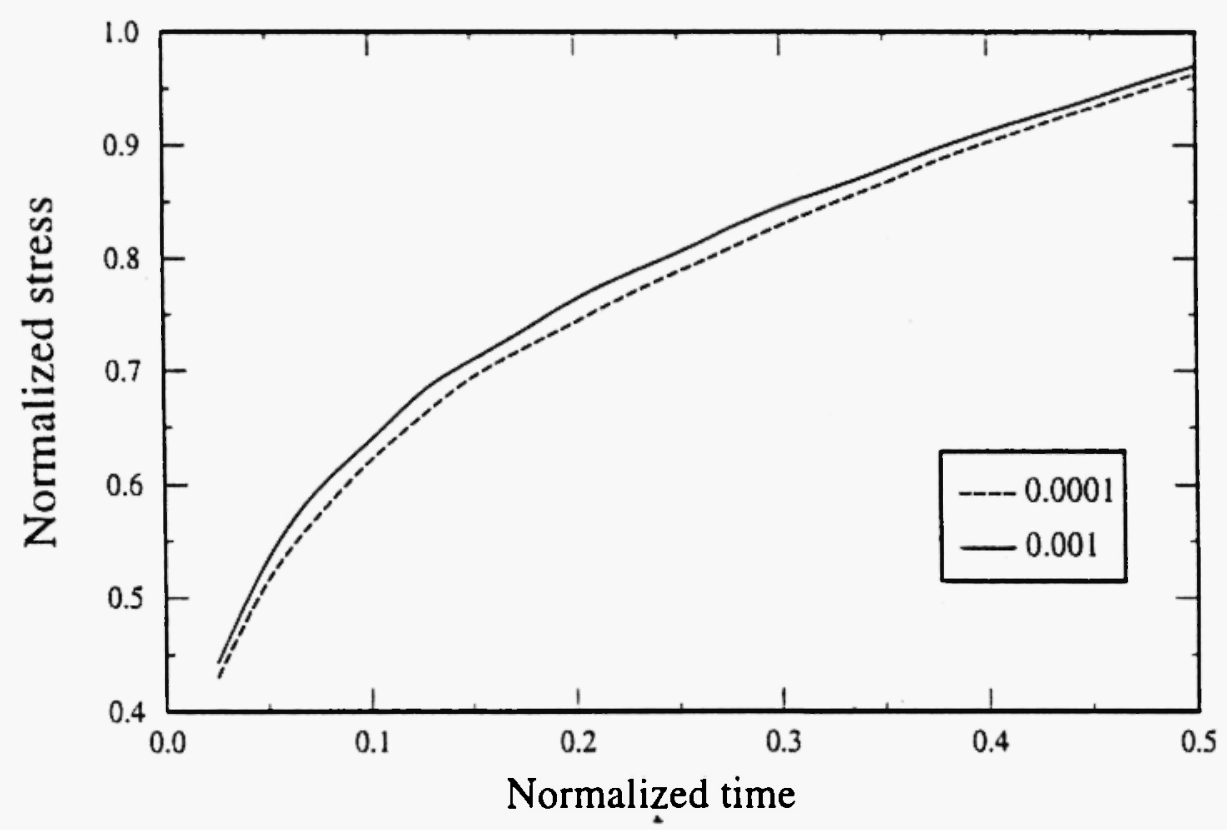

Fig. 8 Effect of the gradient coefficient $C$ on the stress response $(\beta=0.4, C=0.0005)$.

b) Anisotropic softening $(\beta<0)$ : Figure 9 describes the effect of the gradient coefficient for the back stress on the shear band. It also shows that the width of the shear band only depends on the gradient coefficient. However, the stress-strain relation of the material is quite sensitive to the magnitude of the gradient coefficient, especially at the deformation softening regime as shown in Figure 10.

Figure 11 illustrates how the deformation localization varies with deformation. On comparing these results with the case of anisotropic hardening (Figure 7), it is concluded that deformation localization is intensifies due to deformation softening and shear band "healing" does not occur.

Figure 12 discusses the so-called mesh-size sensitivity which is an important factor for assessing the suitability of a theoretical model and associated numerical calculation. Unlike the classical plasticity, the results show clearly that the gradient-dependent plasticity is insensitive to the mesh density. 


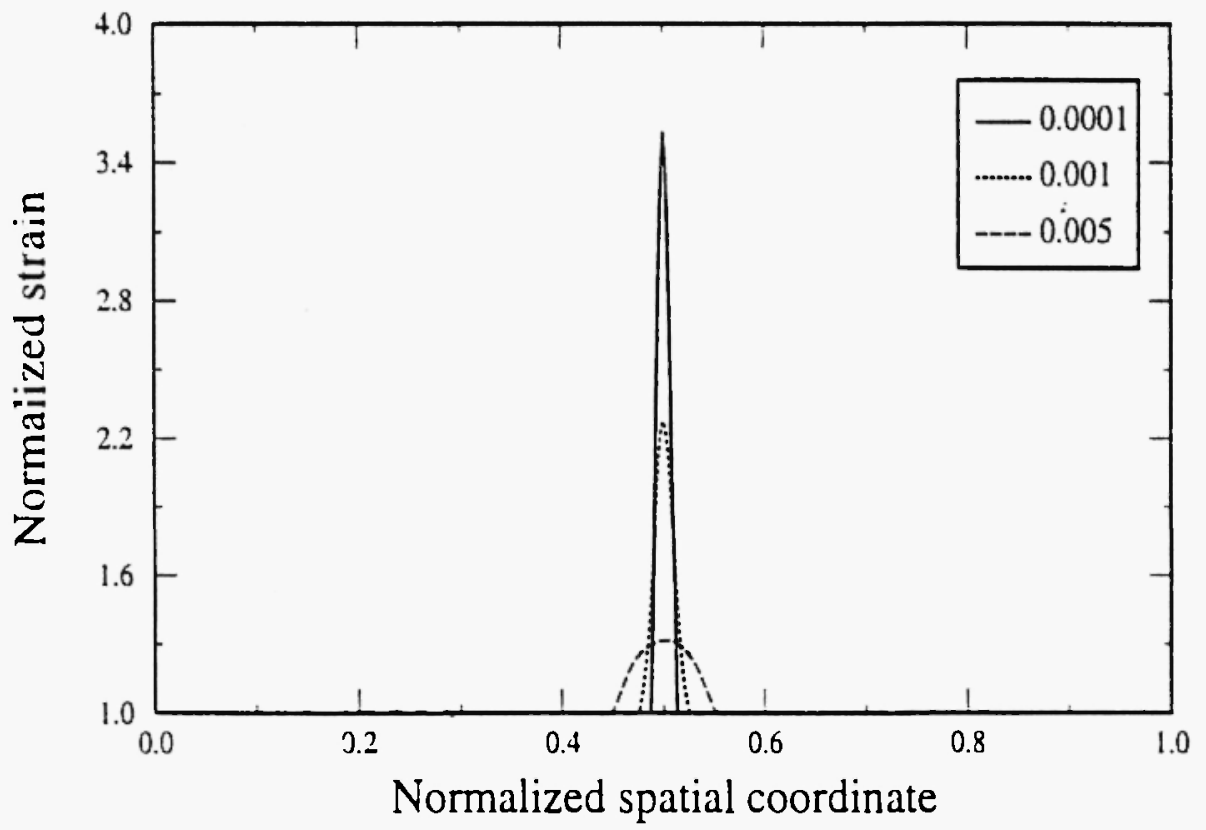

Fig. 9 Effect of the gradient coefficient $\bar{C}$ on the width of shear band. The normalized strain is defined by $\gamma / \gamma_{0}(\beta=-1.0, t=0.2)$.

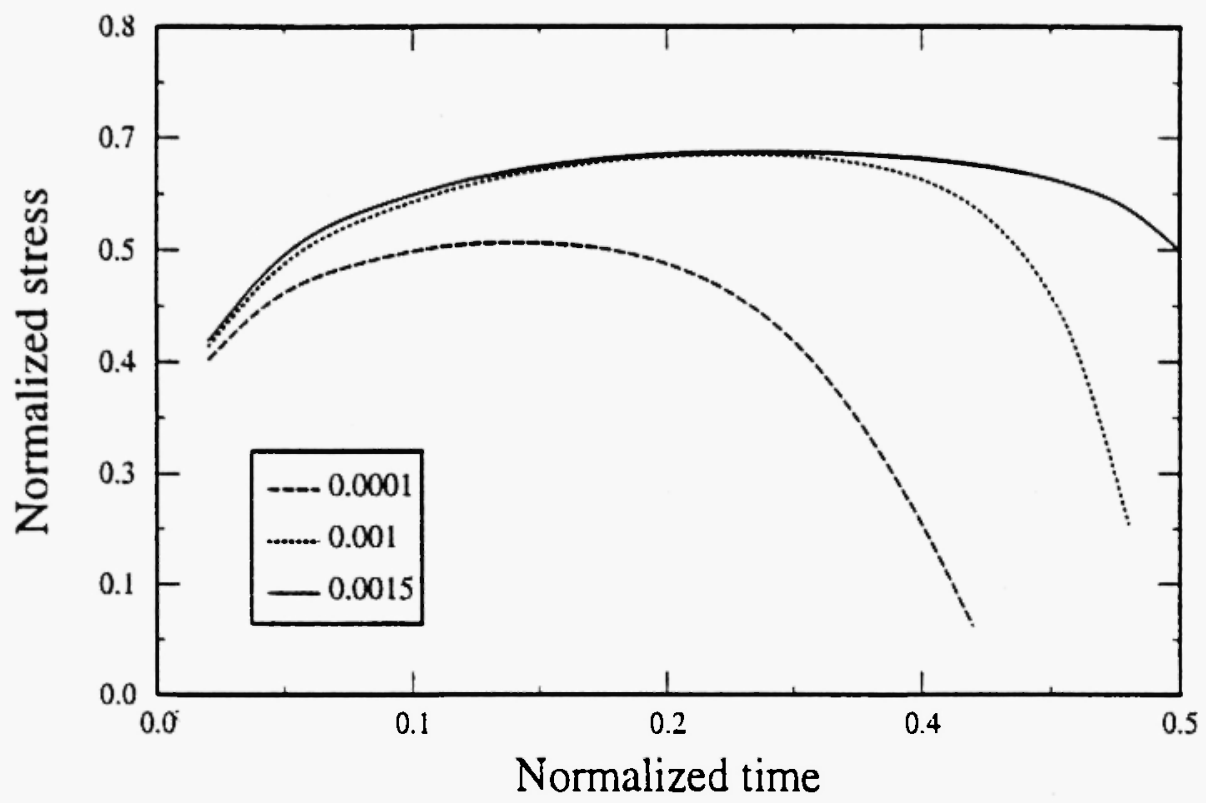

Fig. 10 Effect of the gradient coefficient $\bar{C}$ on the stress response $(\bar{\beta}=-0.8)$. 


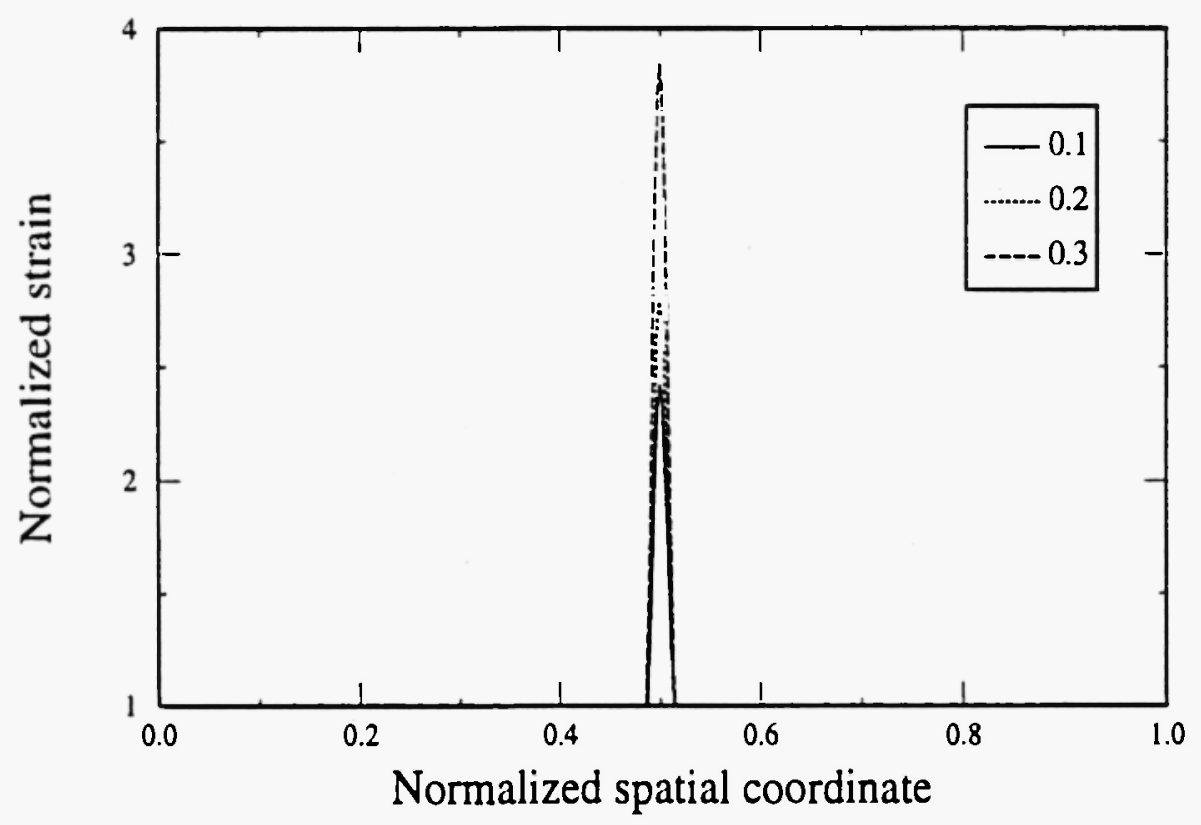

Fig. 11 Evolution of the normalized strain with $t(\bar{\beta}=-0.8, C=0.001)$.

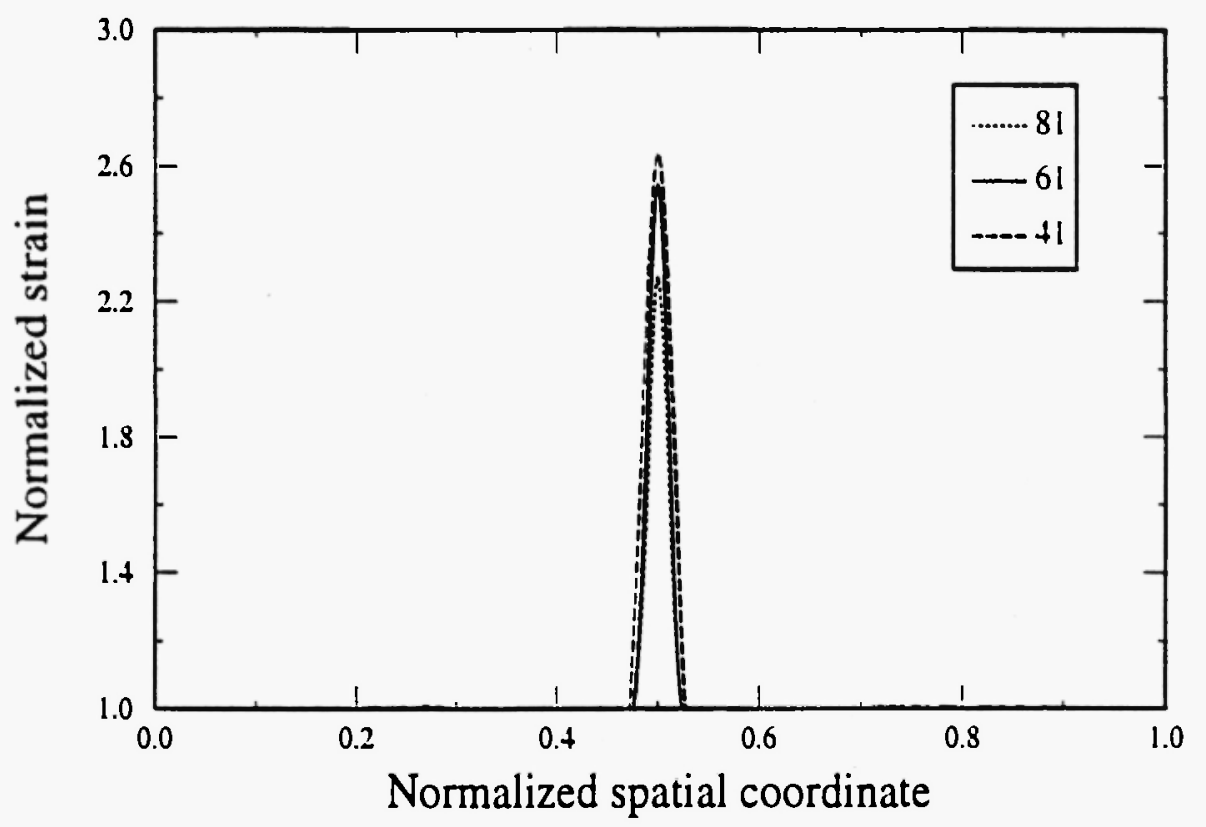

Fig. 12 Mesh-size sensitivity $(\bar{\beta}=-0.8, \bar{C}=0.002$, and $t=0.2)$. 
c) Anisotropic hardening/softening $\left(\bar{\beta}=0.1-5(\gamma-0.1)^{2}\right)$ : In the following, attention is focused on the behavior of anisotropic hardening/softening material where the material parameter $\beta$ may be positive or negative depending on the deformation level. Figure 13 indicates again the dominated influence of the gradient coefficient on the width of shear band. Figure 14 indicates that the stress response of the material is insensitive to the value of the gradient coefficient in the deformation hardening regime, but it is very sensitive to the value of the gradient coefficient in the deformation softening regime.

\subsection{Necking in Porous Materials}

Consider a porous material in uniaxial tension. For the sake of simplicity, the deformation state is assumed to be of the form

$$
\dot{\varepsilon} p_{22}=\dot{r} \dot{c}_{j j}^{n}=-\hat{n} \dot{\varepsilon}_{11}^{n},
$$

where the parameter $\lambda$ is a function of deformation history and regarded as a constant in the analysis below. Following the same steps as those in the above section for the shear banding problem, the governing equations for the present problem read

$$
\begin{gathered}
\rho \frac{\partial v}{\partial t}=\frac{\partial \sigma}{\partial x}, \\
\frac{\partial \varepsilon}{\partial t}=\frac{\partial v}{\partial x}, \\
\frac{\partial \varphi}{\partial t}=(1-\varphi) \Lambda \dot{\varepsilon}^{p}+c \frac{\partial^{2} \varphi}{\partial x^{2}}, \\
\sigma=\sigma_{y} \sqrt{1+(r \varphi)^{2}-2 r c h(\eta \sigma)}
\end{gathered}
$$

where $\eta=1 / \sigma_{b}$ and $\Lambda=1-2 \lambda$. 


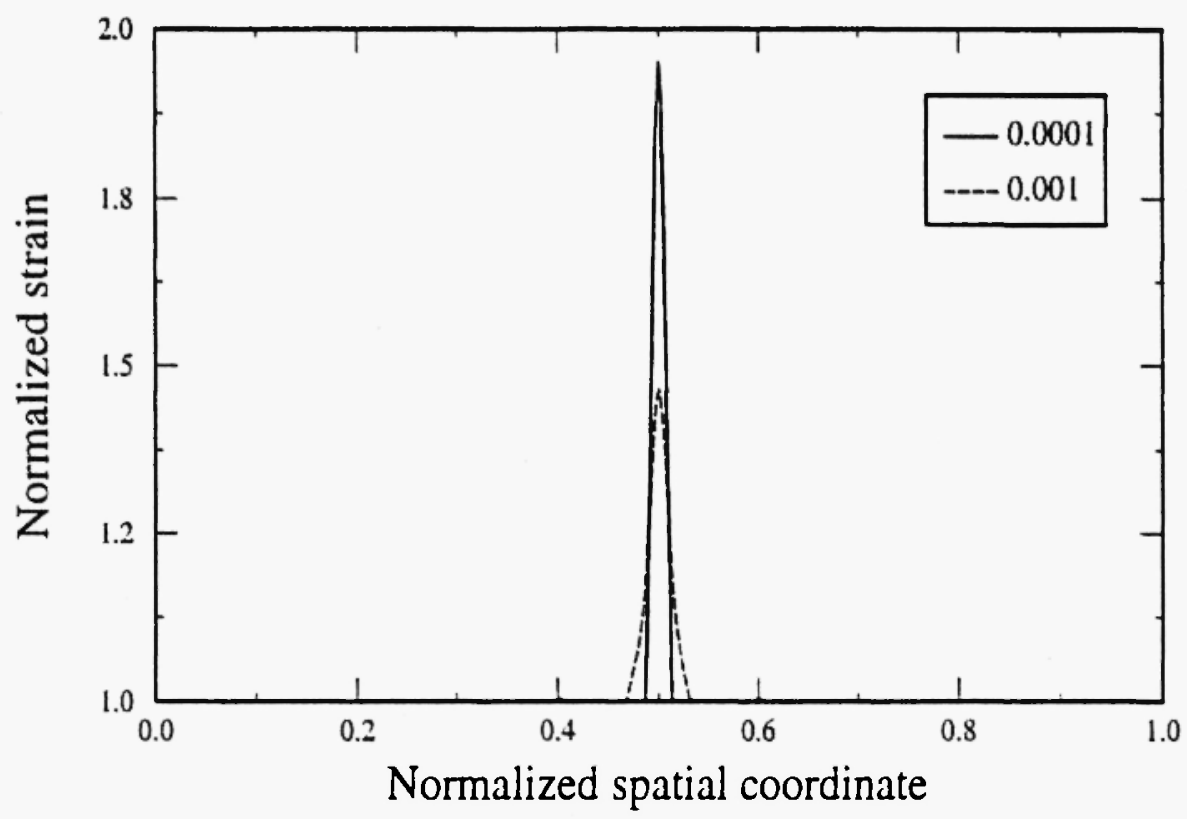

Fig. 13 Effect of gradient coefficient $\bar{C}$ on the width of shear band $(t=0.2)$.

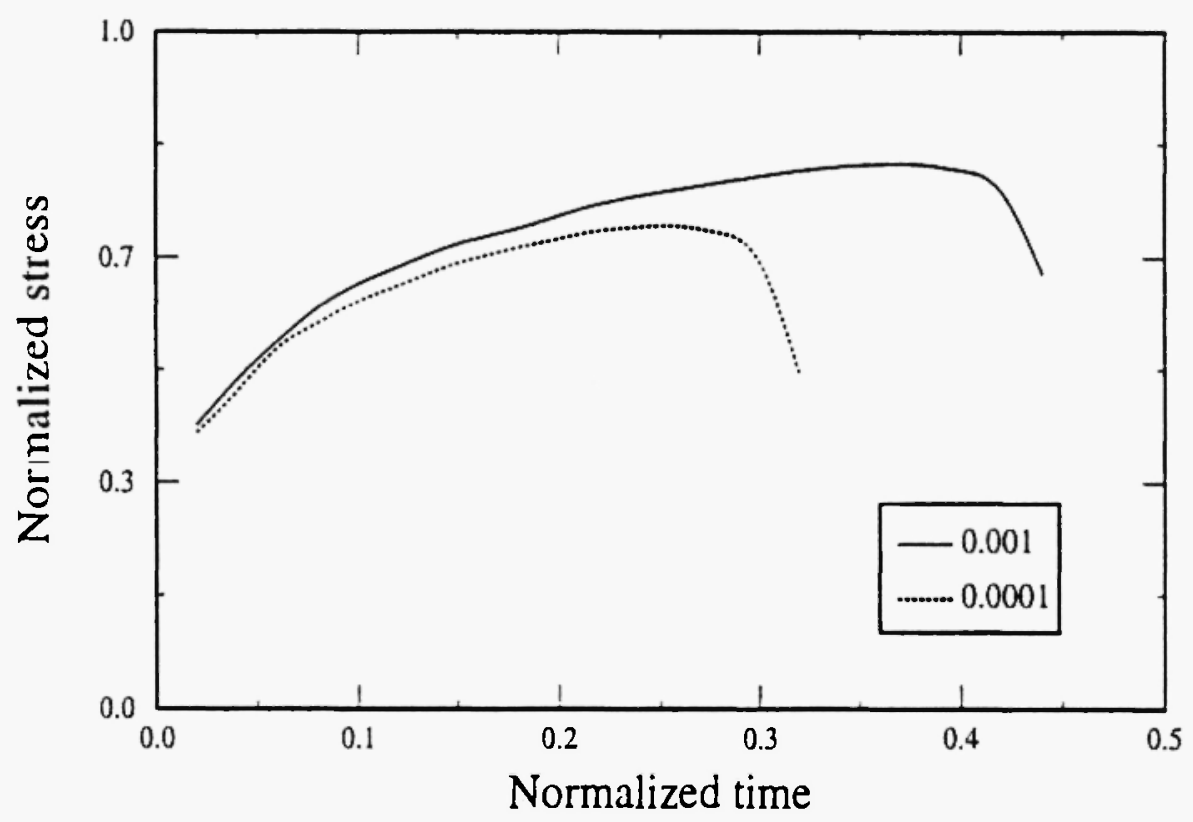

Fig. 14 Effect of gradient coefficient $\bar{C}$ on the stress response. 
The associated boundary and initial conditions are

$$
\begin{gathered}
v=0 \text { and } \frac{\partial \varphi}{\partial x}=0 \quad(\text { for } x=0), \\
v=V . \text { and } \frac{\partial \varphi}{\partial x}=0 \quad(\text { for } x=L), \\
v=V x / L, \varepsilon=0, \varphi=0 \quad(\text { for } t=0) .
\end{gathered}
$$

\section{Linear Stability Analysis}

The characteristic equation resulting from the linear perturbation analysis of eqns. (64)-(67) for the porous material in quasi- static uniaxial tension reads

$$
B \omega+C=0,
$$

with the relevant coefficients defined by

$$
\begin{gathered}
B=Z_{\varepsilon}+Z_{\varphi}(1-\varphi) \Lambda ; \quad C=Z_{\varepsilon} A ; \quad Z_{\varepsilon}=\frac{H \Delta}{\sqrt{\Delta}+\eta r \varphi \cos h(\eta \sigma)}, \\
H=\frac{\partial \sigma_{y}}{\partial \varepsilon} ; \quad Z_{\varphi}=\frac{\sigma_{y}\left(r^{2} \varphi+r \cos h(\eta \sigma)\right)}{\sqrt{\Delta}+\eta r \varphi \cos h(\eta \sigma)} ; \Delta=1+(r \varphi)^{2}-2 r \cos h(\eta \sigma) .
\end{gathered}
$$

The initiation of necking $(\operatorname{Re}(\omega)=0)$ then requires

$$
C=0 \text {. }
$$

This gives the following critical condition for the porosity $\varphi$

$$
\varphi_{c}=\frac{1}{r} \sqrt{2 r \cosh \left(\eta \sigma_{c}\right)-1} .
$$

\section{Non-Linear Numerical Analysis}

Based on the governing equations (64)-(67) and the associated initial and bound- 
ary conditions (68)-(70), a two-step difference scheme is developed to calculate the evolution of localized necking for a porous material. Figure 15 shows the dominant effect of the coefficient of porosity gradient on the width and strength of the necking.

Figure 16 describes the evolution of necking with deformation. It indicates that the width of localized neck does not change for a constant value of the gradient coefficient. Therefore, the neck width depends only on the value of the gradient coefficient, a fact which can be used in experiments to determine the value of this new phenomenological coefficient [20].

Figure 17 gives the stress responses for different values of the gradient coefficient. Because the flow stress is assumed to be a constant in the calculation, the material will soften at the beginning of the deformation due to the evolution of the porosity (damage). The results show that the gradient coefficient also controls the material softening behavior and the corresponding gradient term behaves as a stabilizer for the deformation localization.

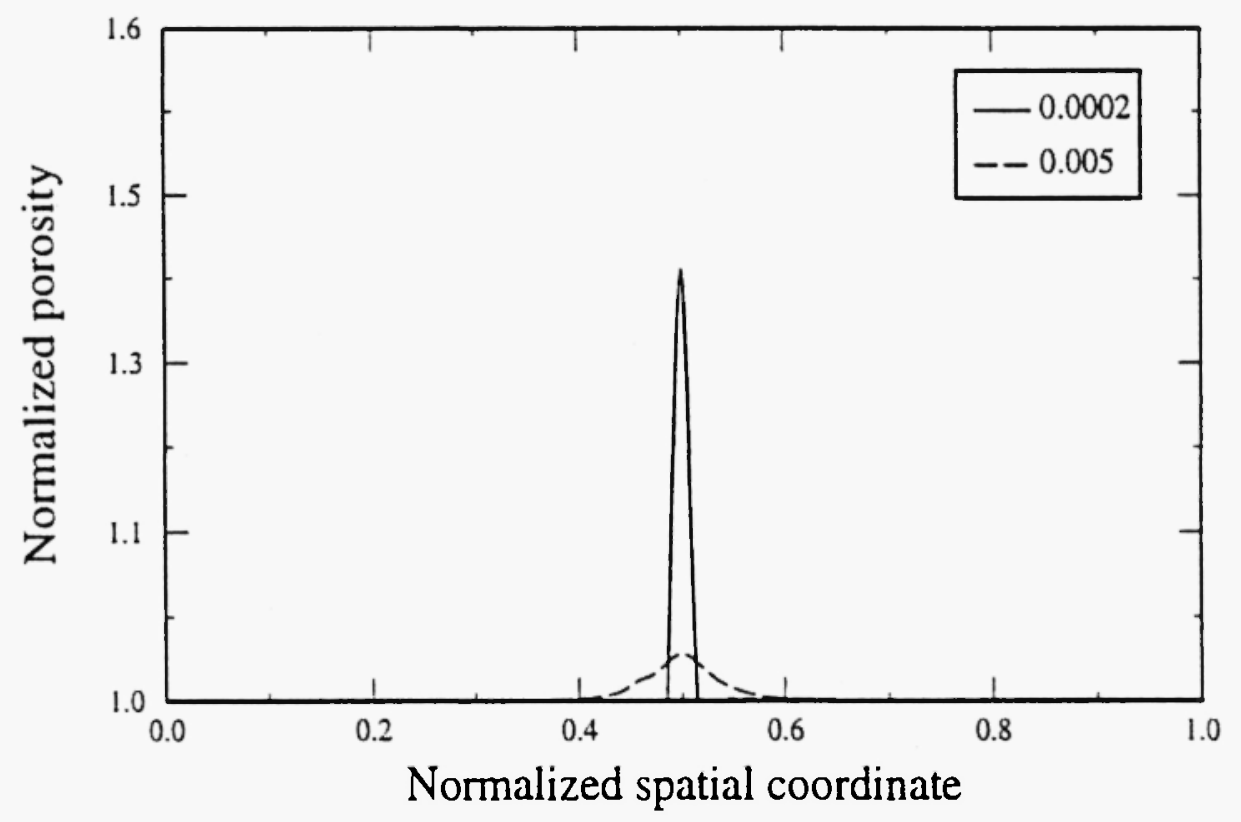

Fig. 15 Effect of gradient coefficient on the structure of the localized necking. 


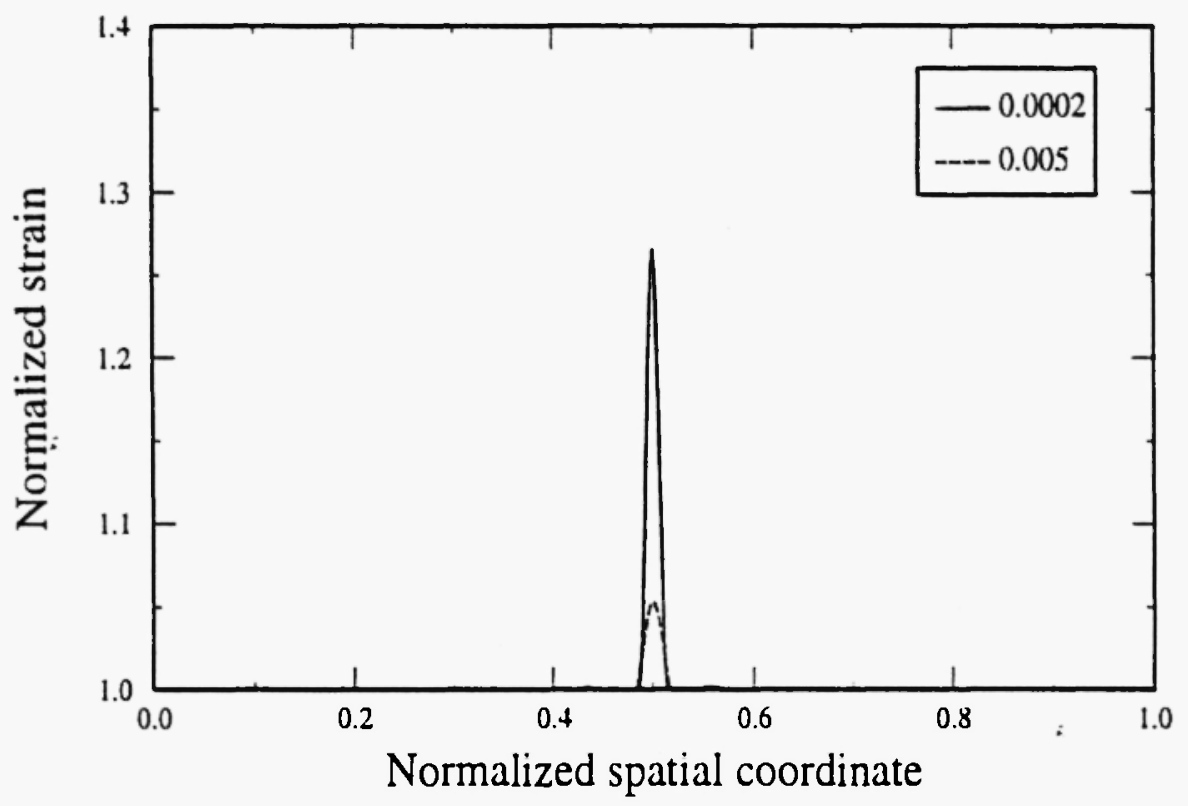

Fig. 16 Evolution of localized necking.

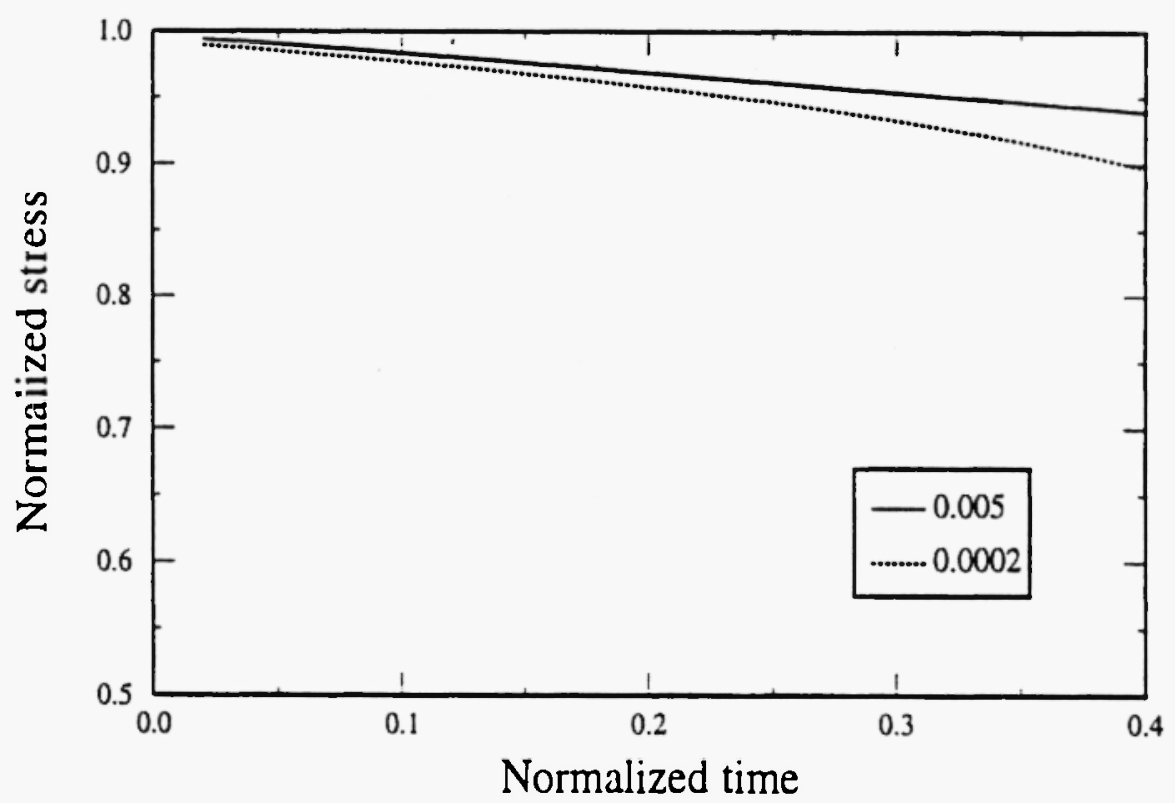

Fig. 17 Stress response for different values of the gradient coefficient. 


\section{References}

[1] E. C. Aifantis, J. Eng. Mat. Tech. 106, 326 (1984).

[2] E. C. Aifantis, Int. J. Plasticity 3, 211 (1987).

[3] E. C. Aifantis, Int. J. Eng. Sci. 30, 1279 (1992).

[4] E. C. Aifantis, Int. J. Eng. Sci. 33, (1995).

[5] H. M. Zbib and E. C. Aifantis, Appl. Mech. Rev. 41, 295(1989).

[6] H. B. Muhlhaus and E. C. Aifantis, Int. J. Solids Struct. 23, 845 (1987).

[7] I. Vardoulakis and E. C. Aifantis, Acta Mech. 87, 197 (1991).

[8] F. Oka, A. Yashima, T. Adachi, and E. C. Aifantis, Appl. Mech. Rev. 45, 103 (1992).

[9] J. Ning and E. C. Aifantis, Unified Constitutive Laws of Plastic Deformation,

K. Krausz and A. Krausz (eds.), Chapter 7, Academic Press (1996).

[10] P. Bridgman, Trans. ASM 32, 553 (1944).

[11] B. D. Coleman and M. L. Hodgdon, Arch. Rat. Mech. Anal. 90, 219 (1985).

[12] H. B. Zbib, Script Met. et Mat. (1995).

[13] E. C. Aifantis and J. B. Serrin, J. Coll. Interf. Sci. 96, 517 (1983).

[14] H. Zbib and E. C. Aifantis, Acta Mech. 92, 209 (1992).

[15] J. W. Hutchinson and K. W. Neale., J. Mech. Phys. Solids 31, 405 (1983).

[16] T. C. Hsu and A. S. Williamson, Proc. Am. Soc. Testing Mats. 65, 575 (1965).

[17] T. C. Hsu, G. S. Littlejohn, and B. M. Marchbank, B., Proc. Am. Soc. Testing Mats. 65, 874 (1964).

[18] F. L. Addessio and J. N. Johnson, J. Appl. Phys. 74, 1640 (1993).

[19] D. Dudzinski and A. Molinari, Int. J. Solids Struct. 27, 601 (1991).

[20] G. L. Panger, Jr., J. L. Ding, H. M. Zbib and E. C. Aifantis, Script Metall. et Mat. 25, 2103 (1991). 
